POPE, B., \& SIEGMAN, A. W. Interviewer specificity and topical focus in relation to interviewee productivity. Journal of Verbal Learning \& Verbal Behavior, 1965, 4, 188-192.

SIEGMAN, A. W., \& POPE, B. Effects of question specificity and anxiety-producing messages on verbal fluency in the initial interview. Journal of Personality \& Social Psychology, 1965, 4, 522-530.

SIEGMAN, A. W., \& POPE, B. The effect of interviewer ambiguity-specificity and topical focus on interviewee vocabulary diversity. Language \& Speech, 1966, 4, 242-249. NOTES

1. The author wishes to thank Mrs. Eileen Entin at the Ohio University Computer Center for her help in analyzing the data.
2. When the time that the Ss looked at the stimulus card was compared, it was found that the HAI group $(\mathrm{N}=24, \overline{\mathrm{X}}=32.6 \mathrm{sec})$ looked significantly longer $[F(1,46)=7.73, p<.01\}$ than the LAI group $(\mathrm{N}=24, \overline{\mathrm{X}}=23.7$.

3. Two Ss were eliminated because they did not produce the minimal criterion of 25 words (HAI-2: $\mathrm{N}=10$ ).

4. A similar analysis was not possible for the 2 -min groups since they produced too few words.

5 . Since the combined mean for Segment 4 $(N=16, \quad \bar{X}=.86)$ and Segment $5 \quad(N=11$, $\bar{X}=.87)$ had $N$ s that were significantly reduced, they were not included in the analysis so as to avoid giving disproportionate weight to their means.

\title{
A note on the "Search after meaning": Sensibleness of paraphrases of well formed and malformed expressions $^{1}$
}

\section{SAMUEL FILLENBAUM, University of North Carolina, Chapel Hill, N.C. 27514}

Subjects were required to assess the sensibleness of sentences where each set of sentences included a source sentence plus some of its paraphrases. Characteristically, the paraphrases of malformed expressions (anomalous sentences, sentences with order inversions, word salads) were judged to be more sensible than their source expressions. This was taken as evidence for $S s^{\prime}$ ability to impose sense on deviant expressions.

It seems reasonable to suppose that a listener will try to make sense of the materials he encounters, and that a malformed expression will, if at all possible, be "interpreted" in such a fashion as to make it intelligible. Linguists have offered various hypotheses to account for this ability to comprehend "semi-sentences" (see, e.g., Chomsky, 1961; Katz, 1964; Ziff, 1964). To the extent that a paraphrase task requires $S$ to understand an utterance in order to construct another one equivalent in meaning, it provides a tool for externalizing that understanding, and may permit us to demonstrate this "search after meaning" in clear fashion. In the course of an exploratory study of paraphrasing, Ss were required to paraphrase both well formed expressions and expressions malformed in various ways. An examination of the paraphrases of the malformed sentences revealed that very often these were "improved," shifting to conventional form. Thus, mistakes in grammar were corrected, words in unconventional order were rearranged, incomplete phrases were completed, anomalous sentences were transformed into rather different but more sensible and intelligible versions, and various sorts of normalizing reshufflings improved word salads. The study to be reported below constitutes an attempt to objectify these impressions by having a new set of Ss rate the sensibleness of the paraphrases as well as that of their source sentences.

\section{METHOD}

We may distinguish two stages in the study that involved (1) obtaining of paraphrases, and (2) rating of the paraphrases and original expressions.

\section{Obtaining the Paraphrases}

A set of 48 sentences was constructed, including well formed expressions as well as semantically anomalous expressions, ungrammatical sentences, sentences with reversals in word order, incomplete sentences, and word salads. These sentences were arranged in random order and given to $40 \mathrm{Ss}$, college undergraduates, with the following instructions:

"Assume that you are trying to communicate each statement or phrase to someone else, and that it is essential that the other person get the sense of it as fully and exactly as possible. Your task is to paraphrase or rephrase each of the 48 items as accurately as you can, conserving every aspect of its meaning as completely as possible. In doing this, you may add or leave out words, you may rearrange or substitute words, as you find necessary."

This procedure yielded 40 paraphrases for each of the 48 source sentences. Next,
20 source sentences were selected in such fashion as to provide both well formed expressions and expressions malformed in various ways. There were seven ordinary, simple, well formed sentences, five semantically anomalous expressions, two expressions involving unusual order in word arrangement, three expressions constituting word salads, and three incomplete sentences. For each of these 20 source sentences, we now constructed two items, where each item consisted of the source sentence plus nine of its paraphrases, the paraphrases being selected and assigned at random to one or the other of the two items. The 10 sentences constituting each item were arranged in random order and typed on a single sheet; two sets of items were then assembled, the order of items being different in each set. Two sets were used, rather than one, to permit assessment of the replicability of the findings. The booklets representing the two item sets were arranged in alternation.

\section{Rating of Source Sentences and Paraphrases}

The Ss, 44 undergraduates of whom 21 responded to Set 1 and 23 to Set 2, were drawn from a population similar to that used in the paraphrase study. They were told:

"Most of the sentences in each set will be quite similar to each other. Your job is to assess the sensibleness or strangeness of the expressions as English (don't worry about the truth or falsity of what is said) For each set of items, read carefully through all 10 items a couple of times, and then pick out the three expressions which are most sensible, well formed, and acceptable as English, and put the letter $A$ against each of these. Also pick out the three expressions which, as English, are the strangest or least acceptable, and put the letter $Z$ against each of these. Remember to make your choice always relative to the 10 iterns in each set."

Given the way ratings were made, one can determine directly for each $S$, on each item, whether or not the source sentence was on the average judged more, as, or less acceptable than its paraphrases. If the source sentence is categorized as $A$, it is more acceptable than at least seven of the nine paraphrases, if it is categorized $Z$, it is less acceptable than at least seven of the nine paraphrases; otherwise, it is as acceptable as the paraphrases. Inspection of the frequencies of $A$ and $Z$ responses to the source sentence will thus indicate the extent to which it was judged more or less acceptable than its paraphrases, and a sign test on these frequencies will permit assessment of the significance of the differences obtained. 
Table 1

Frequency of A (Most Acceptable) and Z (Least Acceptable) Judgments for Source Sentences

\begin{tabular}{|c|c|c|c|c|c|}
\hline & & \multicolumn{2}{|c|}{$\begin{array}{c}\text { Set } 1 \\
(\mathrm{~N}=21)\end{array}$} & \multicolumn{2}{|c|}{$\begin{array}{c}\text { Set } 2 \\
(\mathrm{~N}=23)\end{array}$} \\
\hline & & A & $Z$ & $A$ & Z \\
\hline $\begin{array}{l}\text { Well Formed } \\
\text { Sentences }\end{array}$ & $\begin{array}{r}\text { No. } 1 \\
2 \\
3 \\
4 \\
5 \\
6 \\
7\end{array}$ & $\begin{array}{r}7 \\
8 \\
3 \\
11 \\
17 \\
11 \\
14\end{array}$ & $\begin{array}{l}1 \\
2 \\
4 \\
2^{*} \\
0^{* *} \\
1^{* *} \\
0^{* *}\end{array}$ & $\begin{array}{r}8 \\
7 \\
3 \\
14 \\
7 \\
7 \\
7 \\
10\end{array}$ & $\begin{array}{l}9 \\
3 \\
5 \\
2^{* *} \\
3 \\
1 \\
0^{* *}\end{array}$ \\
\hline $\begin{array}{l}\text { Anomalous } \\
\text { Sentences }\end{array}$ & $\begin{array}{r}\text { No. } 1 \\
2 \\
3 \\
4 \\
5\end{array}$ & $\begin{array}{l}0 \\
3 \\
0 \\
1 \\
4\end{array}$ & $\begin{array}{c}9^{* *} \\
2 \\
19^{* *} \\
11^{* *} \\
6\end{array}$ & $\begin{array}{l}2 \\
0 \\
0 \\
0 \\
5\end{array}$ & $\begin{array}{c}8 \\
18^{* *} \\
23^{* *} \\
16^{* *} \\
5\end{array}$ \\
\hline $\begin{array}{l}\text { Unusual } \\
\text { Word Order }\end{array}$ & No. 1 & $\begin{array}{l}0 \\
0\end{array}$ & $\begin{array}{l}21^{* *} \\
21^{* *}\end{array}$ & $\begin{array}{l}0 \\
0\end{array}$ & $\begin{array}{l}23^{* *} \\
23^{* *}\end{array}$ \\
\hline $\begin{array}{l}\text { Word } \\
\text { Salads }\end{array}$ & $\begin{array}{r}\text { No. } 1 \\
2 \\
3\end{array}$ & $\begin{array}{l}0 \\
0 \\
0\end{array}$ & $\begin{array}{l}20^{* *} \\
21^{* *} \\
21^{* *}\end{array}$ & $\begin{array}{l}0 \\
0 \\
0\end{array}$ & $\begin{array}{l}23^{* *} \\
23^{* *} \\
20^{* *}\end{array}$ \\
\hline $\begin{array}{l}\text { Incomplete } \\
\text { Sentences }\end{array}$ & $\begin{array}{r}\text { No. } 1 \\
2 \\
3\end{array}$ & $\begin{array}{l}0 \\
5 \\
5\end{array}$ & $\begin{array}{l}1 \\
6 \\
2\end{array}$ & $\begin{array}{r}11 \\
6 \\
6 \\
\end{array}$ & $\begin{array}{l}0^{* *} \\
4 \\
1 \\
\end{array}$ \\
\hline
\end{tabular}

$* p<.05 \quad * * \quad p<.01$

\section{RESULTS AND DISCUSSION}

The principal results of the study are shown in Table 1, which presents the frequencies of $A$ and $Z$ responses to the source sentence in each item, for both Sets 1 and 2. It can be seen that when the source sentence is a well formed one in six out of seven cases for Set 1 (four significant) and in five out of seven cases for Set 2 (two significant), it is judged to be more acceptable on the average than its paraphrases. This is reasonable enough if one notes that in paraphrasing such sentences, Ss often elaborated on the original very simple sentences. The results for sentences with words in unusual orders and the results for word salads are very clear. For all five instances in each set, the source sentence is judged to be less acceptable on the average than its paraphrase; all the differences are

significant. Similar results, if not quite so striking, are obtained for the anomalous sentences. For Set 1 , in four out of five cases (three significant), the source sentence is judged less acceptable than its paraphrase; the results for Set 2 are almost identical. There does not seem to be much difference in the acceptability of incomplete source sentences and their paraphrases; in only one case of six is there a significant difference, which is in favor of the incomplete source sentence. ${ }^{2}$

The general tenor of the results is clear and corroborates impressions based on a scrutiny of the paraphrases-malformed source sentences tend to be rephrased in more sensible and acceptable versions, while well formed simple sentences tend, if anything, to be elaborated and made more complex, in large part because that is about the only way in which they can be altered.
Of these two results, the first, of course, is of considerably greater interest and significance insofar as it indicates that understanding is not simply or only understanding of well formed structures, but involves a process that can impose structure and interpretation on expressions that may be syntactically and/or semantically deviant in various ways. A thorough study of the properties of these paraphrases may reveal something as to the nature of the processes involved in understanding and interpretation, and this is perhaps the principal reason for interest in the paraphrase task. Such study will require careful analysis of the nature of the changes occurring under paraphrase in conjunction with a consideration of pertinent linguistic formulations. For some promising beginnings involving analysis of paraphrases of compound nouns, see Gleitman and Gleitman, ${ }^{3}$ which presents results not only of interest in their own right but also for the light they shed on the relation between a transformational grammar as a model of competence and the performance of speakers.

\section{REFERENCES}

CHOMSKY, N. Some methodological remarks on generative grammar. Word, 1961, 17, 219-239.

KATZ, J. J. Semi-sentences. In J. A. Fodor and J, J. Katz (Eds.), The structure of language. Englewood Cliffs, N.J.: Prentice-Hall, 1964.

ZIFF, P. On understanding "understanding utterances." In J. A. Fodor and J. J. Katz (Eds.), The structure of language. Englewood Cliffs, N.J.: Prentice-Hall, 1964.

\section{NOTES}

1. This work was supported in part by PHS Research Grant No. MH 10006 from the National Institute of Mental Health.

2. There were, in addition, four item sets involving ambiguous source sentences. These yielded mixed results. For two cases, the source sentence was judged to be more acceptable than its paraphrases, and for the other two cases, the opposite held true (in both sets).

3. Gleitman, L. R., \& Gleitman, H. Phrase and paraphrase. Unpublished manuscript, 1969. 